Research Paper

\title{
Oxycodone vs. Fentanyl Patient-Controlled Analgesia after Laparoscopic Cholecystectomy
}

\author{
Boo-Young Hwang, Jae-Young Kwon ${ }^{\bowtie}$, Eunsoo Kim, Do-Won Lee, Tae-Kyun Kim, and Hae-Kyu Kim \\ Department of Anesthesia and Pain Medicine, Biomedical Research Institute, Pusan National University Hospital, Busan, Republic of Korea.
}

$\square$ Corresponding author: Jae-Young Kwon, Department of Anesthesia and Pain Medicine, Biomedical Research Institute, Pusan National University Hospital, 179 Gudeok-ro, Seo-gu, Busan 602-793, Republic of Korea. Tel: +82-51-240-7399 Fax: +82-51-242-7466 E-mail: jykwon@pusan.ac.kr.

( ) Ivyspring International Publisher. This is an open-access article distributed under the terms of the Creative Commons License (http://creativecommons.org/ licenses/by-nc-nd/3.0/). Reproduction is permitted for personal, noncommercial use, provided that the article is in whole, unmodified, and properly cited.

Received: 2013.12.10; Accepted: 2014.04.01; Published: 2014.04.30

\begin{abstract}
Objectives Oxycodone is semi-synthetic opioid, oral and parenteral preparations have been widely used for acute and chronic pain. The aim of this study was to assess the efficacy and side effects of oxycodone and fentanyl in patient controlled analgesia (PCA) after laparoscopic cholecystectomy. Methods A prospective, randomized, double-blind study was conducted. 8I patients were randomly divided into two groups; fentanyl (10 mcg fentanyl and $1.5 \mathrm{mg}$ ketorolac) and oxycodone group (I mg oxycodone and I.5 mg ketorolac). After the operation, a blinded observer assessed pain using a numerical rating scale (NRS), infused PCA dose, side effects, sedation levels, and satisfaction.

Results Cumulative PCA dose of oxycodone group at $48 \mathrm{~h}(3 \mathrm{I} .4 \pm 16.0 \mathrm{ml})$ was significantly less than that of fentanyl group $(43.8 \pm 23.1 \mathrm{ml}, P=0.009)$. Oxycodone group showed more nausea at 6 - $24 \mathrm{~h}$ after the operation $(P=0.00 \mathrm{I})$, but there was no difference in satisfaction score $(P=$ 0.073). There were no significant differences in other side effects, sedation and NRS scores between two groups.

Conclusion Oxycodone showed comparable effects for pain relief compared to fentanyl in spite of less cumulative PCA dose. Based on these results, we could conclude that oxycodone may be useful as an alternative to fentanyl for PCA after laparoscopic cholecystectomy.
\end{abstract}

Key words: Fentanyl, Laparoscopic cholecystectomy surgery, Oxycodone, Patient-controlled analgesia, Postoperative pain.

\section{Introduction}

Laparoscopic cholecystectomy surgery is often accompanied by severe pain, although it has advantages of fast recovery, reduced postoperative pain, and short hospital stay [1,2]. Postoperative pain can cause atelectasis, sympathetic over activity, prolonged hospital stay, and decreased patient's satisfaction. Furthermore, unrelieved severe pain may lead to chronic pain. Patient-controlled analgesia (PCA), which allows a patient to administer their own pain relief, is known to provide an efficient way to control postoperative pain [3]. A variety of analgesics including non-steroidal anti-inflammatory drugs
(NSAIDs) and opioids have been used for the PCA. Not only the efficacy of a drug, but also its side effects, duration, and patient's history or general condition are critical factors to be considered in selecting an opioid. The pharmacodynamic and pharmacokinetic effects depend on the type of opioids, route, age, genetic polymorphism, and so on $[4,5]$.

Oxycodone is one of the most widely used opioids for pain management [6,7]. There have been several studies on the efficacy and side effects of oxycodone. Oxycodone has been known to have the same potency with morphine [8]. Many studies 
demonstrated good efficacy of oxycodone on postoperative pain [9-11]. However, most of the previous studies are based on oral oxycodone and bolus intravenous injection of oxycodone. The equivalent dose of oxycodone to fentanyl has not been clarified, especially in PCA.

In this study, we designed a prospective, randomized, and double-blind study to assess the efficacy and side effects of oxycodone PCA compared to those of fentanyl PCA in patients who underwent the laparoscopic cholecystectomy.

\section{Materials and methods}

\section{Study design}

A prospective, randomized and double-blind study was conducted at the Department of Anesthesia and Pain Medicine at Pusan National University Hospital, Korea between June and October 2013. The study protocol was approved by an appropriate institutional review board (IRB), and patients were provided informed consent before a surgery.

\section{Subjects}

90 patients who are ASA class I or II were scheduled for laparoscopic cholecystectomy. Patients of both genders, aged 18 to 65 years old, were selected. Patients, who do not qualify for or do not prefer treatment of opioids or NSAIDs, or who have psychological disorder, chronic pain disorder or preoperative administration of drugs including opioids, antidepressants, gabapentin, pregabalin, and carbamazepine, were excluded. Patients, who cannot use numerical rating scale (NRS), who have increased intracranial pressure, renal failure, hepatic failure, or in pregnancy, were also excluded.

\section{Treatment}

The primary end point was to compare efficacy with oxycodone and ketorolac versus fentanyl and ketorolac. Secondary outcomes were incidences of side-effects and patients' satisfaction. Patients were randomly divided into fentanyl group and oxycodone group. Fentanyl group received $10 \mathrm{mcg}$ fentanyl and $1.5 \mathrm{mg}$ ketorolac, and oxycodone group received $1 \mathrm{mg}$ oxycodone and $1.5 \mathrm{mg}$ ketorolac for a bolus dose. Anesthesia was standardized in both groups. $0.2 \mathrm{mg}$ glycopyrrolate was intramuscularly injected to all the patients $30 \mathrm{~min}$ before induction of anesthesia. After patients arrived in the operating room, their baseline heart rate, mean arterial blood pressure, oxygen saturation were obtained using patient monitor. Bispectral index (BIS, XP version 4.1; Aspect Medical Systems, Newton, MA, USA) monitoring was used to measure the depth of anesthesia. $2 \mathrm{mg} / \mathrm{kg}$ i.v. propofol, $0.6 \mathrm{mg} / \mathrm{kg}$ i.v. rocuronium, and 0.5 $\mathrm{mcg} / \mathrm{kg} / \mathrm{min}$ i.v. remifentanil were administered for the induction, and sevoflurane was used for maintenance. $30 \mathrm{mcg}$ fentanyl/4.5 mg ketorolac or $3 \mathrm{mg}$ oxycodone/4.5 mg ketorolac were administered in the fentanyl or the oxycodone groups, respectively, and an antiemetic was administered $30 \mathrm{~min}$ before the end of surgery. After the operation, $10 \mathrm{mg}$ i.v. pyridostigmine and $0.4 \mathrm{mg}$ i.v. glycopyrrolate were administered, and patients were transferred to post-anesthesia care unit, and stayed until Aldrete score was greater than 8 . Patients received pain control via PCA with a bolus dose of $1 \mathrm{ml}$, a lock-out interval of $6 \mathrm{~min}$, and a four-hour limit of $40 \mathrm{ml}$. If patients complained severe pain above 5, we recommended them to press the button instead of administration of rescue analgesics.

\section{Assessment}

A blinded observer assessed the pain at rest and during coughing based on an NRS, where 0 denotes no pain and 10 denotes worst pain experienced. $\mathrm{Cu}-$ mulative PCA dose and sedation scale were also measured at 1, 4, 8, 12, 24, and $48 \mathrm{~h}$ after the operation. The Ramsay sedation scale was applied to assess the sedation state: 1 = anxious, agitated, or restless; $2=$ cooperative, oriented, and tranquil; 3 = responds to command; $4=$ brisk response to a light glabellar tap or loud auditory stimulus; $5=$ sluggish response to a light glabellar tap or loud auditory stimulus; and $6=$ no response to the stimuli.

Side-effects were recorded at 6, 24, and $48 \mathrm{~h}$ after the surgery. If a patient complained severe nausea, 4 $\mathrm{mg}$ i.v. ondansetron was injected. Patients were asked to rank their satisfaction according to the following scale: 1 = very unsatisfactory; 2 = unsatisfactory; $3=$ neutral; $4=$ satisfactory; $5=$ very satisfactory.

\section{Statistical analysis}

Data are expressed as mean $\pm \mathrm{SD}$. The demographic data were compared using the student's $t$-test. Incidence of side effects was compared between two groups using the chi-square test and Mann-Whitney test. The cumulative PCA dose and the sedation scores were compared using Mann-Whitney test. The NRS were compared using two-way repeated measures ANOVA. The satisfaction scores of two groups were compared using the chi-square test. A probability of $<0.05$ was considered to be significant. SPSS (21.0 IBM statistics data editor SPSS Inc., Chicago, IL, USA) was used for all statistical analyses. An estimated sample size indicated that 41 patients per group would give a $\beta$-risk of $80 \%$ at an a-level of 0.05 for detecting a difference in cumulative PCA dose (35 vs $40 \mathrm{ml}$ ) of at least $5 \mathrm{ml}$ at $48 \mathrm{~h}$ after the operation with a standard deviation of 8.0 for each group in the 
preliminary test. We enrolled 90 subjects for study considering $10 \%$ as exclusion rate.

\section{Results}

90 patients were assessed for the study. 4 patients refused the participation in the study, and 86 patients were included in this study after randomization. A patient of each group was discharged earlier than expected, and 2 patients of fentanyl group and 1 patient of oxycodone group wanted early discontinuation of PCA for emesis.

There was no significant difference between the two groups in the distribution of height, weight, age, and sex (Table 1).

Side-effects such as vomiting, headache, shivering, pruritus, and respiratory depression in the oxycodone group were not significantly different from those in the fentanyl group. However, the patients in the oxycodone group experienced more nausea at 6-24 $\mathrm{h}$ after operation compared to the fentanyl group $(P=$ 0.002 , Table 2).

Table I: Baseline demographic characteristics of patients.

\begin{tabular}{llll}
\hline Characteristics & Fentanyl & Oxycodone & Total \\
\hline Sex & & & \\
Male & 21 & 21 & 42 \\
Female & 19 & 20 & 39 \\
Age, mean years (SD) & $54.4(13.8)$ & $49.6(15.0)$ & $52.0(14.5)$ \\
Weight, mean kg (SD) & $63.6(10.865)$ & $67.8(14.2)$ & $65.7(12.7)$ \\
Height, mean cm (SD) & $164.4(8.909)$ & $164.4(10.4)$ & $164.7(9.6)$ \\
\hline
\end{tabular}

There is no significant difference between groups.

Table 2: Incidence of side effects.

\begin{tabular}{lllll}
\hline Side effects & Fentanyl & Oxycodone & Total & $P$ value \\
\hline Nausea, $n(\%)$ & $14(35)$ & $22(53.7)$ & $36(44.4)$ & 0.091 \\
$<6 \mathrm{~h}$ & $14(35)$ & $18(43.9)$ & $32(39.5)$ & 0.413 \\
$6-24 \mathrm{~h}$ & $5(12.5)$ & $18(43.9)$ & $23(28.4)$ & 0.002 \\
$24 \mathrm{~h}<$ & $4(10)$ & $3(7.3)$ & $7(8.6)$ & 0.667 \\
Vomiting & $0(0)$ & $0(0)$ & $0(0)$ & - \\
Headache, $n(\%)$ & $1(2.5)$ & $2(4.9)$ & $3(3.7)$ & 0.571 \\
Shivering, $n(\%)$ & $0(0)$ & $1(2.4)$ & $1(1.2)$ & 0.320 \\
Pruritus, $n(\%)$ & $3(7.5)$ & $1(2.4)$ & $4(4.9)$ & 0.293 \\
Respiratory depression, $n(\%)$ & $0(0)$ & $0(0)$ & $0(0)$ & - \\
\hline
\end{tabular}

The oxycodone group patients experienced more nausea at 6-24 h after operation compared to the fentanyl group ( $P=0.002$, Chi square test).

The cumulative PCA doses were measured at 1 , $2,4,8,12,24$, and $48 \mathrm{~h}$ after the operation. The oxycodone group showed significantly less amount of cumulative PCA dose compared to the fentanyl group after $8 \mathrm{~h}(P<0.05$, Figure 1$)$. Cumulative PCA dose of oxycodone group at $48 \mathrm{~h}(31.4 \pm 16.0 \mathrm{ml})$ was significantly less than that of fentanyl group $(43.8 \pm 23.1 \mathrm{ml}$, $P=0.009)$. Six patients in fentanyl group and ten patients in oxycodone group who complained severe emesis were administrated ondansetron intravascu- larly. Percentage of severe cases in total nausea of fentanyl group and oxycodone group were $42.9 \%$ and $47.6 \%$. There was no significant difference in administration of ondansetron between both groups.

Sedation levels of the patients were assessed based on the Ramsey sedation scale. The patients in both groups showed similar sedation levels (Figure 2).

There was no significant difference in NRS at rest and during coughing between the two groups and they decreased in process of time from 4 to $48 \mathrm{~h}$ (Figure $3 a$ and $3 b$ ).

There was no significant difference in satisfaction score at $48 \mathrm{~h}$ after the operation $(P=0.073$, Table $3)$.

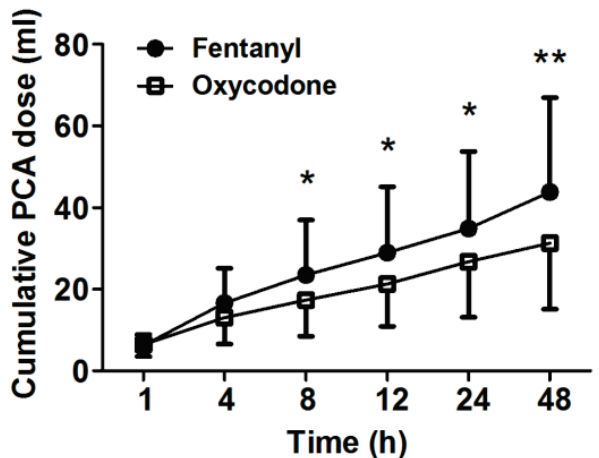

Figure I: Cumulative PCA dose measured at several time points between $\mathrm{I}$ and $48 \mathrm{~h}$ after the operation. The patients of fentanyl group show higher consumption rate than the patients of oxycodone group. Data are expressed as mean $\pm \mathrm{SD}$., $* \mathrm{P}<0.05$ and $* * \mathrm{P}<0.01$ according to Mann-Whitney test.

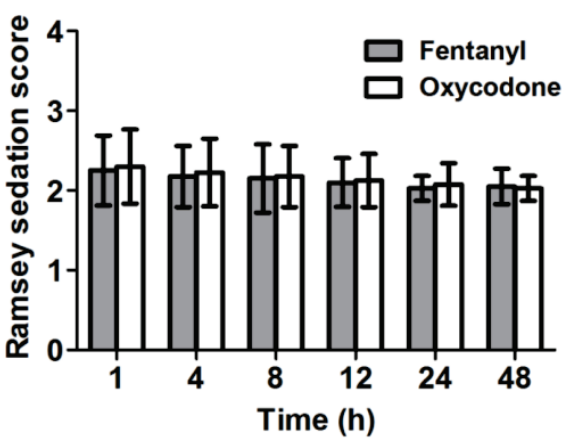

Figure 2: Ramsey sedation scores at several time points between I and 48 $\mathrm{h}$ after the operation. Both groups show similar sedation levels. Data are expressed as mean \pm SD.

Table 3: Satisfaction on pain management at $48 \mathrm{hr}$.

\begin{tabular}{llll}
\hline Satisfaction, $n(\%)$ & Fentanyl & Oxycodone & Total \\
\hline 1 & $0(0)$ & $0(0)$ & $0(0)$ \\
2 & $3(7.5)$ & $0(0)$ & $3(3.7)$ \\
3 & $8(20)$ & $7(17.1)$ & $15(18.5)$ \\
4 & $22(55)$ & $18(43.9)$ & $40(49.4)$ \\
5 & $7(17.5)$ & $16(39)$ & $23(28.4)$ \\
\hline
\end{tabular}

There is no significant difference in satisfaction score at $48 \mathrm{~h}$ after the operation between groups $(P=0.073$, Chi square test). 
(a)

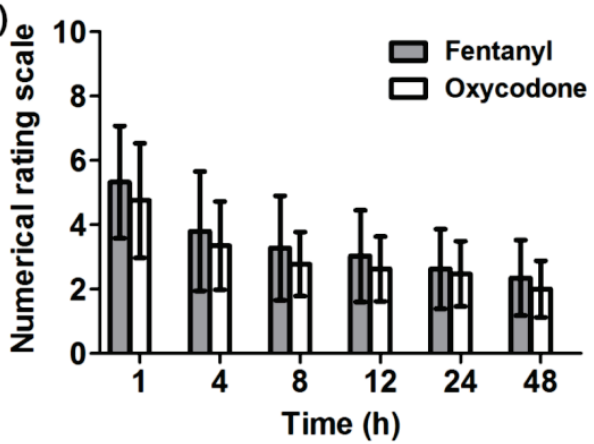

(b)

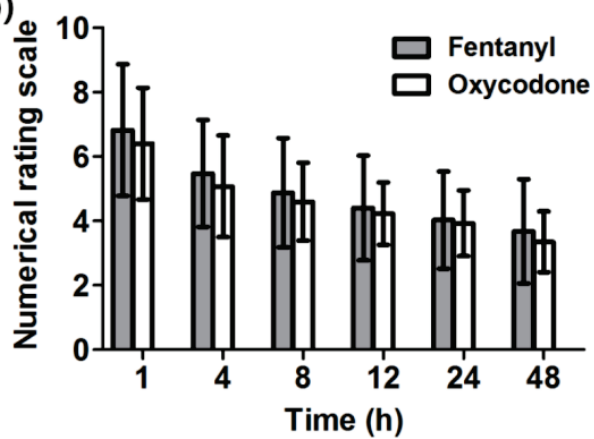

Figure 3: Numerical rating scale (NRS) of pain at I - $48 \mathrm{~h}$ after the operation. The NRS of pain intensity was assessed (a) at rest and (b) during coughing. There is no significant difference between both groups. Both groups show marked decrease in pain as the time passed. Data are expressed as mean \pm SD.

\section{Discussion}

There have been many studies comparing the effect of different opioids on acute post-operative pain. No previous study has compared oxycodone and fentanyl in PCA. We showed that oxycodone group needed less cumulative PCA doses compared to fentanyl group, while analgesic effects were not different.

Oxycodone is a semi-synthetic opioid and oral oxycodone has been widely used for treatment for postoperative pain, chronic pain, and pain due to malignant disease [6, 7]. Parenteral oxycodone appears to be equipotent with morphine [8]. Morphine has a 1:80-100 potency ratio compared to fentanyl [12]. In this study, however, the oxycodone group showed significantly less cumulative PCA dose to maintain the NRS values comparable with that of the fentanyl group. Mean cumulative PCA dose of the oxycodone group was $31.4 \mathrm{ml}$ at $48 \mathrm{~h}$ after operation, while that of the fentanyl group was $43.8 \mathrm{ml}$. Therefore, we could suggest that the potency ratio of fentanyl to oxycodone might be around 1:75 and oxycodone might be more potent than morphine (4:3). Similarly, there have been several literatures supporting that oxycodone is more potent than morphine. Lenz et al. [10] have suggested that the equianalgesic efficacy of oxycodone is 1.6 times higher than that of morphine. It has been reported that oxycodone provides better analgesia but also more side effects on bolus injection [11]. It has also been known that oxycodone have more effective to visceral pain than other opioids [13]. Kalso et a 1. [14] observed that less oxycodone was needed for the pain relief than morphine.

It is too early to conclude that oxycodone of $1 \mathrm{mg}$ is more potent or effective than fentanyl $100 \mathrm{mcg}$ or morphine $1 \mathrm{mg}$. We should consider other factors such as distribution, clearance, bioavailability, and duration of the drugs. Kalso et al. [14] also suggested that the parenteral oxycodone provided fast and long-lasting pain relief as compared to morphine. Oxycodone has a slightly longer duration of action when compared with fentanyl ( $t_{1 / 2}: 4$ h 52 min vs. $3 \mathrm{~h}$ $39 \mathrm{~min}$ ). The analgesic effect of a single dose of i.v. fentanyl may be shorter because of redistribution [11]. In this study, the similar analgesic potency was noted during very early postoperative period. Effect of the opioid duration on the PCA consumption is uncertain, because we did not evaluate the time to the first bolus injection after administrating loading dose. There is a possibility that the longer action duration of oxycodone compared to fentanyl saved the cumulative consumption of opioids.

The pain intensity of both groups decreased as time went on, thus the cumulative opioid dose also decreased. This result implies that the pain management during the acute phase is critical. Severe acute pain may lead to chronic pain. Therefore, preemptive and aggressive postoperative multimodal analgesia is required [15]. Most patients complained of pain during acute phase, and the NRS of the pain intensity was over 5 at $1 \mathrm{~h}$ after the operation. Initial bolus doses of oxycodone and fentanyl group were $3 \mathrm{mg}$ and $30 \mathrm{mcg}$ in this study. Therefore, we suggest that bolus dose should be increased or another rescue drugs are necessary in the acute period.

Most nausea occurred within $24 \mathrm{~h}$, and it decreased as the time passed. There was no difference in nausea occurrence until $6 \mathrm{~h}$ after operation. The nausea before $6 \mathrm{~h}$ might be due to inhalation agents, laparoscopic surgery, severe acute pain, gastroparesis, and so on [16]. The incidence rate of nausea at 6-24 $\mathrm{h}$ after operation in the oxycodone group was higher than that in the fentanyl group although the cumulative PCA dose in the oxycodone group was lower than that in the fentanyl group. Similar to our results, Pedersen et al. [17] demonstrated that morphine and oxycodone produced similar analgesia after percutaneous kidney stone surgery, but the nausea occurred more frequently in the treatment of oxycodone compared to morphine. Low doses of opioids activate mu opioid receptors in the chemoreceptor trigger zone (CTZ), thereby stimulating vomiting. Oxycodone and 
fentanyl share mu opioid receptor [18]. We guess that the longer duration and larger dose administration for bolus injection in oxycodone group induced more frequent nausea.

In this study, fifty percents of all patients showed nausea, so it seems to require a routine administration of antiemetics or more careful management of the PCA setting. This result was unexpected that the satisfaction of oxycodone group was similar with fentanyl group although the incidence of nausea was higher in oxycodone group. It might be due to longer duration and higher efficacy for one bolus dose in oxycodone, and nausea subsided quickly by $4 \mathrm{mg}$ i.v. ondansetron. This result implies that oxycodone could be useful for PCA to manage postoperative pain.

In this study, ketorolac $(1.5 \mathrm{mg} / \mathrm{ml}$ in concentration) was mixed to PCA solution. Ketorolac has been known to reduce opioid consumption and opioid related side effects [19]. Ketorolac -induced side effects such as increased bleeding tendency and renal toxicity did not occur in this limited number of patients. Maximum cumulative dose of ketorolac were $150 \mathrm{mg}$ and $133 \mathrm{mg}$ in fentanyl and oxycodone group for 48 hrs and did not exceed daily recommended dosage.

In this study, the cumulative PCA dose does not mean how many times patients press the button, because we adjusted the lock-out interval as $6 \mathrm{~min}$. If we allowed the patients to shorten lock-out interval, the differences between the cumulative PCA doses of two groups might be widen. In addition, the target of this study was healthy adults in both genders, thus the effects of bioavailability, metabolism, genetic polymorphism, underlying disease, and type of operations have not been investigated. Recent study suggests that there is a significant interindividual variation in the need for oxycodone for sufficient analgesia [20]. Therefore, further evaluation and study about oxycodone for PCA should be conducted.

Oxycodone showed comparable effects for pain relief compared to fentanyl in spite of less cumulative PCA dose. Based on these results, we could conclude that oxycodone may be useful as an alternative to fentanyl for PCA after laparoscopic cholecystectomy.

\section{Acknowledgements}

This study was supported by a grant of the Korean Heath Technology R\&D Project, Ministry of Health and Welfare, Republic of Korea. (A070001).

\section{Competing Interests}

The authors have declared that no competing interest exists.

\section{References}

1. Chan HS, Ha XF, Ooi PJ, et al. A prospective comparative study between conventional and laparoscopic cholecystectomy. Singapore Med J. 1995; 36: 406-9.

2. Yilmaz H, Arun O, Apiliogullari S, et al. Effect of laparoscopic cholecystectomy techniques on postoperative pain: a prospective randomized study. J Korean Surg Soc. 2013; 85: 149-53.

3. Hudcova J, McNicol E, Quah C, et al. Patient controlled opioid analgesia versus conventional opioid analgesia for postoperative pain. Cochrane Database Syst Rev. 2006; 18: CD003348.

4. Pergolizzi J, Böger RH, Budd K, et al. Opioids and the management of chronic severe pain in the elderly: consensus statement of an International Expert Panel with focus on the six clinically most often used World Health Organization Step III opioids (buprenorphine, fentanyl, hydromorphone, methadone, morphine, oxycodone). Pain Pract. 2008; 8: 287-313.

5. Ing Lorenzini K, Daali Y, Dayer P, et al. Pharmacokinetic-pharmacodynamic modelling of opioids in healthy human volunteers. a minireview. Basic Clin Pharmacol Toxicol. 2012; 110: 219-26.

6. Poyhia R. Opioids in anaesthesia: a questionnaire survey in Finland. Eur J Anaesthesiol 1994; 11: 221-30.

7. Kokki H, Kokki M, Sjovall S. Oxycodone for the treatment of postoperative pain. Expert Opin Pharmacother 2012; 13: 1045-58.

8. Silvasti M, Rosenberg P, Seppala T, et al. Comparison of analgesic efficacy of oxycodone and morphine in postoperative intravenous patient-controlled analgesia. Acta anesthesiol Scand. 1998; 42: 576-80.

9. Blumenthal S, Min K, Marquardt M, et al. Postoperative intravenous morphine consumption, pain scores, and side effects with perioperative oral controlled-release oxycodone after lumbar discectomy. Anesth Analg. 2007; 105: 233-7.

10. Lenz H, Sandvik L, Qvigstad E, et al. A comparison of intravenous oxycodone and intravenous morphine in subject-controlled postoperative analgesia after laparoscopic hysterectomy. Anesth Analg. 2009; 109: 1279-83.

11. Koch S, Ahlburg P, Spangsberg N, et al. Oxycodone vs. fentanyl in the treatment of early post-operative pain after laparoscopic cholecystectomy: a randomised double-blind study. Acta Anaesthesiol Scand. 2008; 52: 845-50.

12. Pereira J, Lawlor P, Vigano A, et al. Equianalgesic dose ratios for opioids: a critical review and proposals for long-term dosing. J Pain Symptom Manage. 2001; 22: 672-87.

13. Staahl C, Dimcevski G, Andersen SD, et al. Differential effect of opioids in patients with chronic pancreatitis: an experimental pain study. Scand J Gastroenterol. 2007; 42: 383-90.

14. Kalso E, Pöyhiä R, Onnela $\mathrm{P}$, et al. Intravenous morphine and oxycodone for pain after abdominal surgery. Acta Anaesthesiol Scand. 1991; 35: 642-46.

15. Kehlet H, Jensen TS, Woolf CJ. Persistent postsurgical pain: risk factors and prevention. Lancet. 2006; 367: 1618-25.

16. Watcha MF, White PF. Postoperative nausea and vomiting. Its etiology, treatment, and prevention. Anesthesiology. 1992; 77: 162-84.

17. Pedersen KV, Olesen AE, Drewes AM, et al. Morphine versus oxycodone analgesia after percutaneous kidney stone surgery. Urolithiasis. 2013; 41: 423-30.

18. Smith HS, Laufer A. Opioid induced nausea and vomiting. Eur J Pharmacol. 2014; 722: 67-78.

19. Picard P, Bazin JE, Conio N, et al. Ketorolac potentiates morphine in postoperative patient-controlled analgesia. Pain 1997; 73: 401-6.

20. Kokki M, Broms S, Eskelinen M, et al. Analgesic concentrations of oxycodone --a prospective clinical PK/PD study in patients with laparoscopic cholecystectomy. Basic Clin Pharmacol Toxicol. 2012; 110: 469-75. 